Diabetologe 2007 · 3:184-191

DOI 10.1007/s11428-007-0129-z

Online publiziert: 12. April 2007

๑) Springer Medizin Verlag 2007

S. Bilz $\cdot$ U. Keller

Klinik für Endokrinologie, Diabetologie und Klinische Ernährung,

Universitätsspital Basel

\title{
Fettleber und Lipidstoffwechsel
}

Die Prävalenz in der Gesamtbevölke-

Die nichtalkoholische Fettlebererkrankung ist ein Merkmal des metabolischen Syndroms. Sie zeichnet sich durch eine Akkumulation von Triglyzeriden in der Leber aus, die auf verschiedensten Ursachen beruht. Neben vermehrter Produktion und vermindertem Abbau von Fetten spielen auch deren Zufuhr über Nahrungsmittel, Störungen in verschiedenen Stoffwechselwegen sowie angeborene und erworbene Erkrankungen des Fettstoffwechsels eine Rolle.

NAFLD - eine wichtige Komponente des metabolischen Syndroms

Die nichtalkoholische Fettlebererkrankung (,nonalcoholic fatty liver disease“, NAFLD) ist als Lebererkrankung definiert, die durch eine makrovesikuläre Leberverfettung gekennzeichnet und nicht durch übermäßigen Alkoholkonsum bedingt ist [1]. Ebenso müssen alternative Ätiologien, wie Virushepatitiden, ausgeschlossen werden. Das Spektrum der Erkrankung umfasst eine einfache Fetteinlagerung, d. h. eine hepatische Fettdeposition ohne begleitende entzündliche Vorgänge, ebenso wie progressive Manifestationen, die durch zusätzliche entzündliche Infiltrate (nichtalkoholische Steatohepatitis, NASH) und fibrotischen Umbau bis zur Zirrhose gekennzeichnet sind. Die Bedeutung der NAFLD beruht insbesondere auch darauf, dass sie eine wichtige $\mathrm{Ma}$ nifestation endemischer Stoffwechselerkrankungen wie der Adipositas, des Typ2-Diabetes und des assoziierten metabolischen Syndroms darstellt. rung wird mit 10-24\% beziffert, bei Übergewichtigen beträgt sie $55-75 \%$.

In der Pathogenese der Erkrankung wird der Insulinresistenz, die mit einem erhöhten Fluss freier Fettsäuren zur Leber sowie einer gesteigerten hepatischen Denovo-Lipogenese, also der Triglyzeridneusynthese aus Kohlenhydratvorstufen, einhergeht, eine große Bedeutung zugeschrieben [25]. Am Beginn der Erkrankung steht nachgewiesenermaßen die Triglyzeridakkumulation in der Leber.

Im Folgenden werden die pathophysiologischen Veränderungen des Lipidstoffwechsels und die Begleitmerkmale, die zu einer Lebersteatose führen können und somit Wegbereiter der NAFLD und ihrer Folgeerkrankungen sind, beschrieben.

\section{Herkunft des Fetts in der Leber}

Eine vermehrte hepatische Triglyzeridablagerung kann prinzipiell reultieren aus:

- einem vermehrten Angebot freier

Fettsäuren an die Leber,

- einer gesteigerten Triglyzeridneusynthese im Rahmen der De-novo-Lipogenese oder

- einer vermehrten hepatischen Aufnahme triglyzeridreicher Lipoproteine.

Auch eine Störung des Exports hepatischer Lipoproteine kann mit einer Triglyzeridakkumulation in der Leber einhergehen (• Abb. 1).

Neue Erkenntnisse klinischer Studien, die auf der kombinierten Anwendung von stabilen Isotopen und Leberbiopsien beruhten, ergaben, dass bei übergewichtigen
Patienten mit einer NAFLD etwa 59\% der hepatischen Triglyzeride aus wiederveresterten freien Fettsäuren entstehen, $26 \%$ im Rahmen der De-novo-Lipogenese neu gebildet werden und die verbleibenden $15 \%$ Nahrungstriglyzeriden entsprechen, die mit triglyzeridreichen Remnants rezeptorvermittelt aufgenommen werden (• Abb. 2) [10].

$\checkmark$ Die hepatische Lipoproteinproduktion wird v. a. durch das Substratangebot bestimmt

Interessanterweise zeigte die Analyse der Herkunft der Triglyzeride in den hepatisch sezernierten Very-low-Density-Lipoproteinen (VLDL) eine gleichartige Verteilung, was mit der Ansicht übereinstimmt, dass die hepatische Lipoproteinproduktion v. a. durch das Substratangebot, also durch hepatische Triglyzeride, und in geringerem Ausmaß auch durch Cholesterinester bestimmt wird (• Abb. 2) [13].

\section{Freie Fettsäuren}

Die Assoziation erhöhter Konzentrationen freier Fettsäuren mit der Insulinresistenz ist seit langem bekannt und auch bei Patienten mit NAFLD nachgewiesen [25]. Pathophysiologisch liegt die Insulinresistenz der Lipolyse zugrunde, d. h., dass die Fettsäurenfreisetzung aus Adipozyten durch Insulin nicht adäquat supprimiert wird. Auch proinflammatorische Zytokine wie TNFa und Interleukin 6 (IL-6), deren Produktion und Konzentration bei Patienten mit metabolischem Syndrom erhöht sind, steigern die Lipolyse in Adipozyten [30]. Die quantitative Extraktion freier Fettsäuren aus dem portalvenösen 
Hier steht eine Anzeige.

算 Springer 


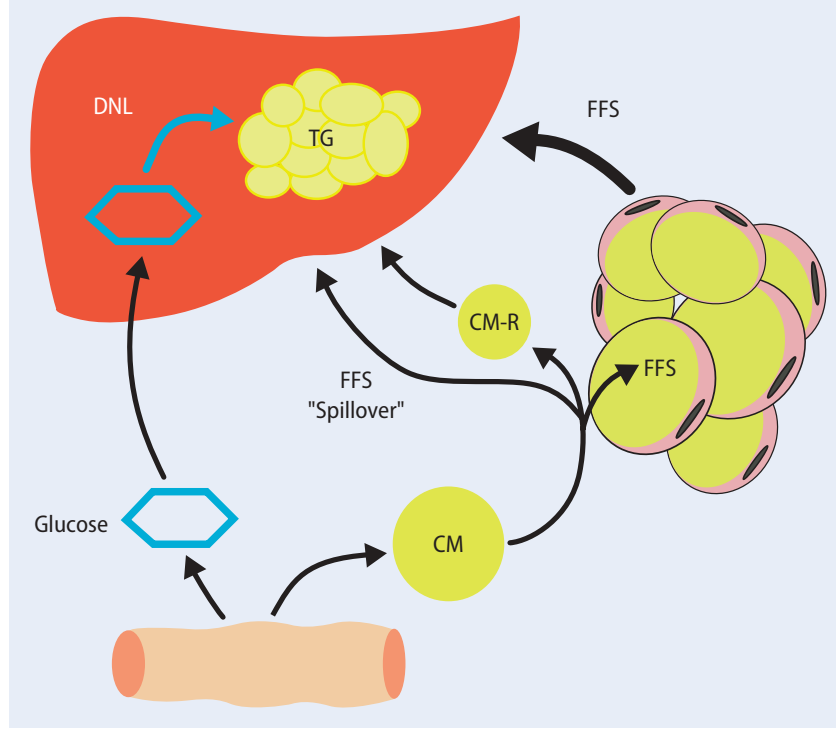

Abb. $1<$ Herkunft der in der Leber bei einer NAFLD vermehrt abgelagerten Triglyzeride, FFS freie Fettsäuren; $C M$ Chylomikronen; $C M-R$ ChylomikronenRemnants; DNL Denovo-Lipogenese; TG Triglyzeride

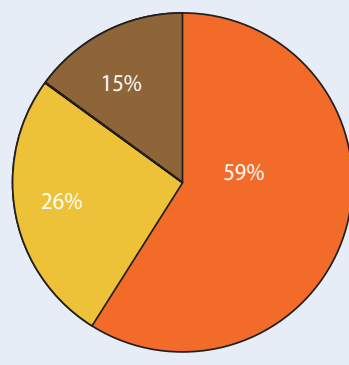

Leber-Triglyceride

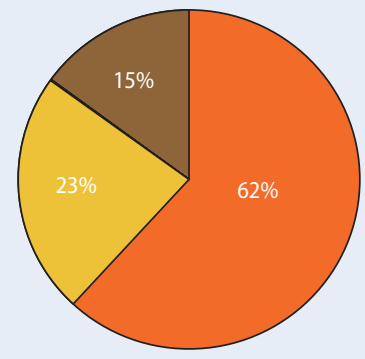

VLDL-Triglyceride
Freie Fettsäuren De novo Lipogenese Nahrungsfett

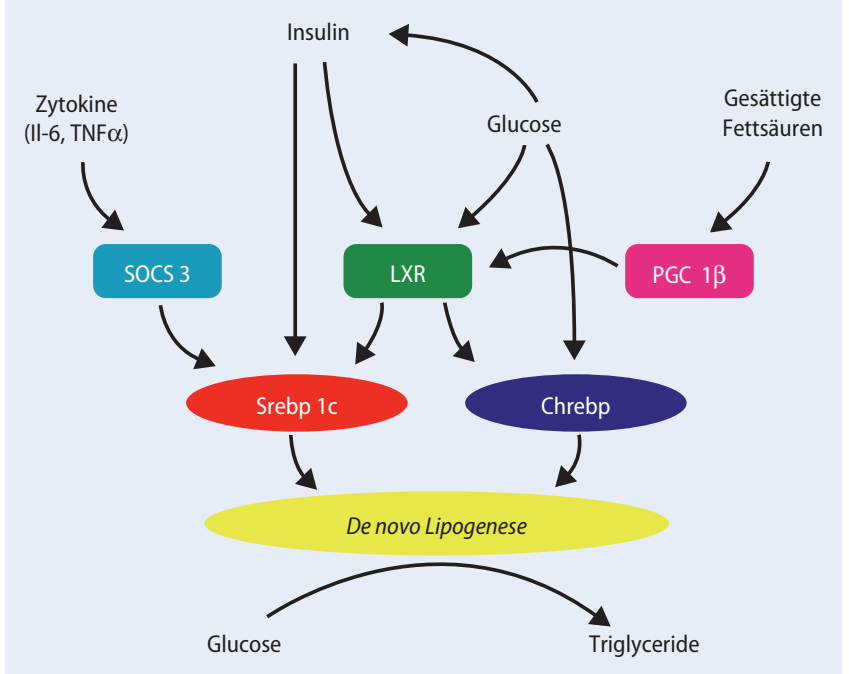

Abb. $2<$ Quantitative Zusammensetzung der Leber- und VLDL-Triglyzeride bei Patienten mit NAFDL nach ihrer Herkunft

Abb. $3<$ Molekulare
Grundlagen der ge-
steigerten De-novo-Li-
pogenese in verschie-
denen Lebersteatose-
modellen, Erklärung
und Abkürzungen
s. Text

Abb. $3<$ Molekulare lagen der gepogenese in verschiedenen Lebersteatoseund Abkürzungen s. Text

tiert [13]. Ebenso bestimmt die hepatische Verfügbarkeit dieser beiden unpolaren Lipide, die durch das mikrosomale Triglyzeridtransferprotein (MTTP) auf Apolipoprotein B10o, das strukturell wichtigste Apoprotein der VLDL, übertragen werden, die VLDL-Sekretionsrate. Als Folge geht die NAFLD oft mit einer gesteigerten Triglyzeridproduktion und Hypertriglyzeridämie einher [13].

\section{Kohlenhydrate}

Der 2. bedeutende Stoffwechselweg, der zur hepatischen Fettakkumulation führen kann, ist die hepatische De-novo-Lipogenese (DNL), also die Umwandlung von Glukose in Fettsäuren, die dann zu Triglyzeriden verestert werden.

\section{(> Die gesteigerte De-novo- Triglyzeridsynthese aus Kohlenhydraten ist pathogenetisch wichtig}

Blut ist nicht limitiert, sodass der Fettsäurenfluss aus dem subkutanen und v. a. dem viszeralen Fettdepot bestimmend für die hepatische Fettsäureaufnahme ist. In Übereinstimmung hiermit konnte bei übergewichtigen Männern mit einer Lebersteatose eine direkte Korrelation zwischen dem Ausmaß der Steatose und der
Konzentration freier Fettsäuren während eines oralen Glukosetoleranztests nachgewiesen werden [14].

In der Leber werden die Fettsäuren oxidiert oder hauptsächlich zu Triglyzeriden, weniger zu Cholesterinestern, verestert. Diese werden in der Leber abgelagert oder in Form von Lipoproteinen expor-
Resultate neuerer Studien zeigten, dass Erkrankungen, die durch eine Insulinresistenz und somit in der Regel auch durch eine NAFLD gekennzeichnet sind, oft mit einer gesteigerten DNL einhergehen [9]. Dies stellt eigentlich ein Paradox dar, da die DNL einen durch Insulin stark stimulierten Vorgang darstellt, der hepatische und periphere Glukosestoffwechsel von Patienten mit metabolischem Syndrom aber insulinresistent sind. Somit liegt hier eine so genannte „gemischte hepatische Insulinresistenz" vor: Einerseits kann die hepatische Glukoseproduktion durch Insulin nicht adäquat supprimiert werden, andererseits steigert die aus der Insulinresistenz resultierende Hyperinsulinämie die immer noch insulinempfindliche DNL, sodass ein Circulus vitiosus vorliegt. Dieses Phänomen ist durch molekularbiologische und tierexperimentelle Untersuchungen gut belegt [23].

\section{Rolle von Srebp 1c und Chrebp}

In klassischen insulinresistenten Mausmodellen, die auch eine Lebersteatose zeigen, ist der wichtigste Transkriptionsfaktor der DNL, das Srebp ic („,sterol-regulatory-element-binding protein 1c") hochreguliert, obwohl gleichzeitig die Signalübertragung 
vom Insulinrezeptor $\mathrm{zu}$ wichtigen Signalmolekülen des hepatischen Glukosestoffwechsels vermindert ist $[17,23]$.

Das Substrat der DNL, Glukose selbst, kann unabhängig von seiner Wirkung auf die Insulinsekretion über die Aktivierung des nukleären Rezeptors LXR („liver X receptor") einerseits Srebp 1c, andererseits das „carbohydrate response element binding protein“ (Chrebp), welches ebenfalls ein wichtiger Regulator der Glykolyse und DNL ist, hochregulieren und die hepatische Triglyzeridneusynthese steigern [4, $6,18]$. Die Bedeutung von Chrebp als pathogenetischer Faktor in der Entstehung der NAFLD wird dadurch unterstrichen, das die Downregulation der Chrebp-messenger-RNA in der Leber von leptindefizienten ob/ob-Mäusen, einem wichtigen NAFLD-Modell, die Lebersteatose und die hepatische Insulinresistenz verbessert (- Abb. 3) [8].

\section{Srebp 1c und Chrebp sind an der Regulation der De- novo-Lipogenese beteiligt}

Auch die überhöhte Zufuhr von Fruktose, die einen Bestandteil des Haushaltszuckers (Saccharose) darstellt und vielfach als Süßungsmittel eingesetzt wird (fruktosereicher Maissirup), führt zumindest im Tiermodell über eine Steigerung der DNL zu einer NAFLD [2]. Zudem können proinflammatorische Zytokine wie TNFa über $\mathrm{SOCS}_{3}$ („suppressor of cytokine signaling $3^{\text {") }}$ und Srebp 1c die DNL stimulieren und so eine Lebersteatose und Insulinresistenz hervorrufen (• Abb. 3) [24]. Schließlich konnte auch bei Patienten mit einer NAFLD in der Leber eine gesteigerte Expression von Genen der DNL nachgewiesen werden [19].

\section{Adiponektin und NAFLD}

Als potenziell wichtiger pathogenetischer Faktor in der Entstehung der NAFLD wurde das Fettgewebehormon Adiponektin identifiziert, dessen Konzentration bei Patienten mit NAFLD erniedrigt ist [3]. Erniedrigte Adiponektinwerte sind mit Insulinresistenz assoziiert, und die Behandlung mit Adiponektin führt in lipodystrophen Mausmodellen zu einer Abnahme der Lebersteatose [28].

Diabetologe 2007· 3:184-191 DOI 10.1007/s11428-007-0129-z

C) Springer Medizin Verlag 2007

S. Bilz $\cdot$ U. Keller

Fettleber und Lipidstoffwechsel

Zusammenfassung

Die hepatozelluläre Anreicherung von Triglyzeriden ist das wichtigste und auch initiale Kennzeichen einer Gruppe von Erkrankungen, die unter dem Begriff "nichtalkoholische Fettlebererkrankung" zusammengefasst und als Merkmal des metabolischen Syndroms erkannt wurden. Die Insulinresistenz bei diesem Syndrom betrifft auch das Fettgewebe, weshalb die Lipolyse gesteigert ist; zudem sind die Masse v. a. von viszeralem Fett und die Serumkonzentration lipolytisch wirksamer Zytokine erhöht. All diese Faktoren steigern die Plasmakonzentrationen von freien Fettsäuren, was zur Fettanlagerung in der Leber Anlass gibt. Weiterhin werden durch die begleitende Hyperinsulinämie

die De-novo-Lipogenese stimuliert und damit vermehrt Kohlenhydrate in Fette umgewandelt. Zudem gelangen mit zirkulierenden Lipoproteinen Triglyzeride in die Leber. In den letzten Jahren konnten wichtige molekulare Mechanismen, die der Dysregulation des Glukose- und Fettstoffwechsels beim metabolischen Syndrom zugrunde liegen und zur hepatischen Triglyzeridablagerung führen, identifiziert werden.

\section{Schlüsselwörter}

Nichtalkoholische Fettlebererkrankung · Metabolisches Syndrom · Hepatozelluläre Triglyzeride - Lipidmetabolismus · Glukosemetabolismus

\section{Fatty liver disease and lipid metabolism}

\section{Abstract}

Nonalcoholic fatty liver disease is characterized by hepatocellular triglyceride accumulation and has been identified as a novel feature of metabolic syndrome. Both peripheral and hepatic insulin resistance are important pathogenetic traits. Free fatty acids released from adipose tissue due to enhanced lipolysis are the quantitatively most important triglyceride precursors. The hyperinsulinemia associated with insulin resistance promotes de novo hepatic lipogenesis from carbohydrate molecules. Circulating triglyceride rich lipoproteins are a further source of hepatic fat. A network of transcription factors and molecular mechanisms resulting in dysregulation of hepatic glucose and lipid metabolism have been characterized and are briefly discussed.

\section{Keywords}

Nonalcoholic fatty liver disease · Metabolic syndrome · Hepatocellular triglycerides · Lipid metabolism · Glucose metabolism 


\section{Leitthema}

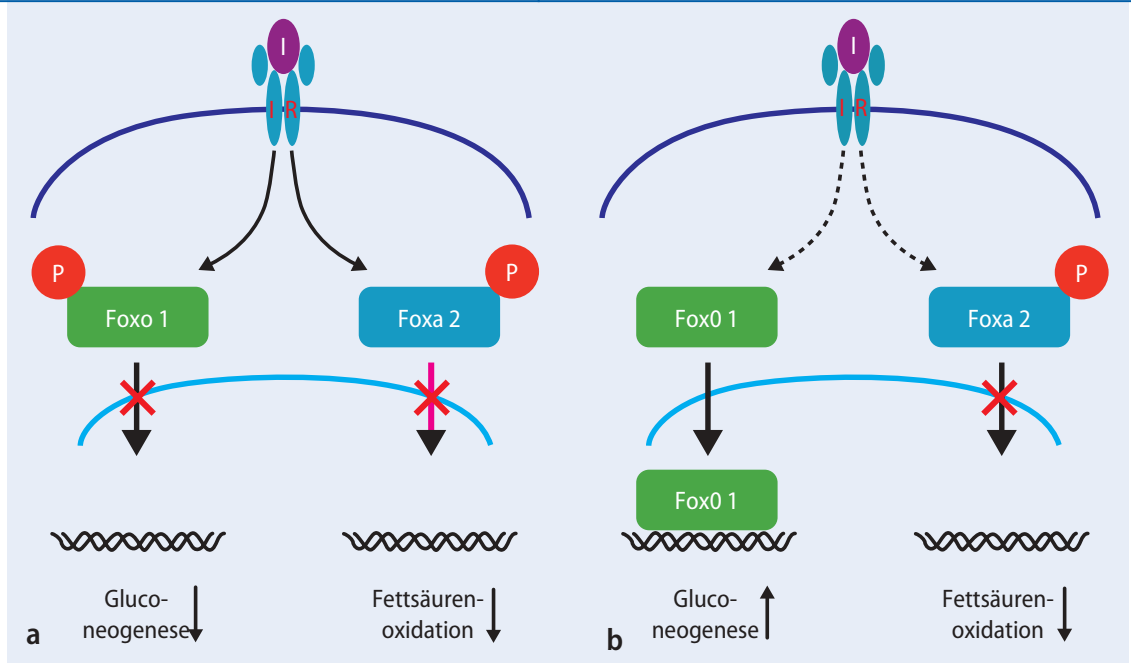

Abb. $4 \Delta$ Rolle von Foxo1 und Foxa2 in der Regulation der hepatischen Glukoseproduktion und Fettsäureoxidation bei Insulinresistenz, I Insulin, IR Insulinrezeptor

Adiponektin wirkt zumindest $\mathrm{z}$. T. über die Aktivierung der AMP-Kinase, welche wiederum die Acetyl-CoA-Carboxylase, das Schrittmacherenzym der DNL, hemmt [29].

\section{Foxo1 und Foxa2 - wichtige Regulatoren der Glukoneogenese und Fettsäurenoxidation}

Als weitere wichtige Signalmoleküle, die die gemischte hepatische Insulinresistenz unterhalten, konnten die „forkhead transcription factors" Foxo1 und Foxa2 identifiziert werden. Sie werden nach Bindung von Insulin an seinen Rezeptor und Aktivierung des Insulinsignalwegs phosphoryliert, was zur Folge hat, dass sie nicht aus dem Zytoplasma in den Nukleus gelangen können, um dort die Genexpression zu modulieren (• Abb. 4 a).

Die Abschwächung des Insulinsignals bei Insulinresistenz führt dazu, dass Foxo1 nicht phosphoryliert wird. Es kann folglich in den Zellkern wandern und dort Gene der Glukoneogenese aktivieren, wodurch die hepatische Glukoseproduktion gesteigert wird. Foxa2, ein Regulator der Fettsäurenoxidation, ist „insulinempfindlicher" und wird trotz des abgeschwächten Insulinsignals phosphoryliert und im Zytoplasma festgehalten. Die Fettsäurenoxidation wird nicht aktiviert, was ebenfalls zur hepatischen Triglyzeridakkumulation beiträgt ( $\mathbf{A b b}$. 4b) [27].

\section{Nahrungsfett und Fettleber}

Eine wichtige Rolle in der Entstehung der NAFLD spielt auch das zugeführte Nahrungsfett. In Triglyzeriden veresterte Fettsäuren aus intestinal sezernierten Chylomikronen können im Rahmen der Lipolyse in peripheren Geweben durch einen Prozess, der als "spillover" bezeichnet wird, in den Pool zirkulierender freier Fettsäuren und somit zur Leber gelangen. Ebenso werden triglyzeridreiche Chylomikronen-Remnants rezeptorvermittelt in die Leber aufgenommen ( $\bullet$ Abb. 1) [10].

Im Tiermodell kann bereits nach 3-tägiger Zufuhr einer fettreichen Diät eine NAFLD induziert werden. Hierbei gleicht das Fettsäurenprofil in der Leber der Ratten jenem des zugeführten Nahrungsfetts. Interessanterweise tritt in diesem Experiment früh eine isolierte hepatische Insulinresistenz bei noch normaler peripherer Insulinsensitivität auf. Dies weist darauf hin, dass in diesem Modell die NAFLD nicht Folge der peripheren Insulinresistenz und der daraus resultierenden Hyperinsulinämie ist, sondern die gesteigerte Fettzufuhr primär zu einer Lebersteatose führt, welche dann die Insulinresistenz begünstigt [21]. Neue, experimentelle Daten ergaben zudem, dass gesättigte Fettsäuren die DNL über die Aktivierung der Transkriptionsfaktoren PGC-1 $\beta$, (peroxisome proliferator activated receptor coactivator $1 \beta^{\prime \prime)}$, LXR und Srebp 1c induzieren können, sodass trotz einer erhöhten exogenen Fettzufuhr noch eine vermehrte hepatische Fettsynthese induziert wird (• Abb. 3) [15].

\section{Nahrungscholesterin - Progression der NAFLD zur NASH}

In neueren tierexperimentellen Untersuchungen wurde gefunden, dass eine exzessive diätetische Cholesterinzufuhr zu einer mitochondrialen Cholesterinüberladung führt, die die Sensitivität der Hepatozyten gegenüber der zytotoxischen und proinflammatorischen Wirkung von LPS und TNFa deutlich erhöht und die Bildung freier Sauerstoffradikale fördert.

\section{— Die Cholesterinüberladung \\ könnte somit ein Teilfaktor sein, der den Übergang einer simplen Lebersteatose in eine Steatohepatitis bedingt.}

Sie hat eine Depletion des wichtigen Radikalfängers Glutathion zur Folge. Entsprechend konnte in diesem Experiment die Repletion der Hepatozyten mit Glutathion das Auftreten einer TNFa-induzierten Steatohepatitis verhindern. Auch in den Lebern von ob/ob-Mäusen konnten eine erhöhte mitochondriale Konzentration freien Cholesterins und erniedrigte Glutathionspiegel nachgewiesen werden. Interessanterweise wurden nachfolgend durch eine Therapie mit dem Cholesterinsynthesehemmer Atorvastatin die freie Cholesterinkonzentration in den hepatischen Mitochondrien gesenkt, die mitochondriale Glutathionkonzentration angehoben und die Steatohepatitis verbessert [16].

\section{NAFLD außerhalb des metabolischen Syndroms}

\section{Lipodystrophien}

Alle bislang beschriebenen Mechanismen stellen Charakteristika der mit dem metabolischen Syndrom assoziierten NAFLD dar. Daneben gibt es weitere Lipidstoffwechselstörungen, die scheinbar paradoxerweise das Auftreten einer NAFLD begünstigen.

Lipodystrophien sind angeborene oder erworbene Erkrankungen, die durch eine Umverteilung von subkutanem Fettge- 


\section{Hier steht eine Anzeige.}

黛 Springer 
webe der Extremitäten und des Gesichts an den Stamm, nach intraabdominal und auch in die Leber und Skelettmuskulatur gekennzeichnet sind. Die HIV-Infektion - bzw. die Therapie mit einer hochaktiven antiretroviralen Therapie (HAART) - stellt die bedeutendste sekundäre Form der Lipodystrophien dar.

- Gemeinsam ist den erworbenen und genetisch bedingten Formen, dass eine Störung der Adipogenese ein wichtiger pathogenetischer Faktor ist.

Mutationen der Gene der AGPAT (Acylglyzerolphosphat-Acyl-Transferase), einem wichtigen Enzym der Triglyzeridsynthese, des PPAR $\gamma$ (Peroxisomenproliferator-aktivierter-Rezeptor $\gamma$ ), dem bedeutendsten Transkriptionsfaktor in der Regulation der Adipogenese und der Lamine, deren Funktion in der Adipogenese nicht letztlich geklärt ist, konnten als molekulare Grundlage von Lipodystrophien identifiziert werden. Die ektope Fettdeposition in diesen insulinempfindlichen Organen ist oft mit einer massiven Insulinresistenz assoziiert [12].

\section{Primäre Hypertriglyzeridämien}

Die familiär kombinierte Hyperlipidämie (FCHL) und die familiäre $\mathrm{Hy}$ pertriglyzeridämie (FHTG) sind relativ prävalente (1:200-500) primäre Hypertriglyzeridämien, deren molekulare Grundlagen nicht geklärt sind. Wie beim metabolischen Syndrom führt auch bei diesen Erkrankungen ein vermehrtes hepatisches Substratangebot (Triglyzeride, Cholesterinester) zur VLDL- und somit Triglyzeridüberproduktion. Patienten mit einer FCHL sind oft auch insulinresistent.

\section{Auch primäre Hypertri- glyzeridämien sind mit einer Fettleber assoziiert}

Eigene, unveröffentlichte Untersuchungen zeigten, dass Betroffene häufig eine Lebersteatose aufweisen. Bei Patienten mit FHTG konnte interessanterweise eine verminderte ileale Gallensäurenresorption nachgewiesen werden [11]. Der hepatische Gallensäurenrezeptor (FXR, Far-
nesoid-X-Rezeptor) ist ein wichtiger negativer Regulator der De-novo-Lipogenese, sodass ein verminderter Gallensäurenfluss zur Leber möglicherweise zu einer gesteigerten DNL und somit einer Fettleber führt [26]. Umgekehrt resultiert die Aktivierung von FXR mit Chenodeoxycholsäure, welche ein potenter FXR-Agonist ist, in einer verminderten DNL und hepatischen Triglyzeridproduktion [2].

\section{Hepatischer Triglyzeridexport und Fettleber}

Nicht nur eine vermehrte hepatische Triglyzeridproduktion, sondern auch eine Störung des Lipidexports kann mit einer NAFLD einhergehen. Dies wird durch den Nachweis einer Lebersteatose bei Patienten mit familiären Hypobetalipoproteinämien, von denen ein Teil auf Mutationen im Apolipoprotein-B10o-Gen beruht, unterstützt [22].

Auch bei der Abetalipoproteinämie, deren molekulare Grundlage Mutationen im mikrosomalen Triglyzeridtransferprotein (MTTP) sind, wurden Zeichen einer Leberverfettung gefunden [20]. Dies wird durch aktuelle Daten untermauert, die nachwiesen, dass auch die pharmakologische Hemmung des MTTP zu einer Leberverfettung führt [7]. Schließlich konnte auch bei nicht selektionierten Patienten mit einer NAFLD eine verminderte hepatische Apo-B-Produktion als Hinweis für die wichtige Rolle des Lipidexports in der Genese der Erkrankung gefunden werden [5].

\section{Fazit für die Praxis}

Aus dem Fettgewebe freigesetzte Fettsäuren, im Rahmen der De-novo-Lipogenese synthetisierte sowie mit RemnantLipoproteinen aufgenommene Triglyzeride können in der Leber zu einer vermehrten Fettdeposition führen und Wegbereiter der NAFLD sein. Die periphere Insulinresistenz, die mit einem erhöhten Fettsäurenfluss aus dem Fettgewebe einhergeht, die resultierende Hyperinsulinämie sowie erhöhte Konzentrationen proinflammatorischer Zytokine, die v. a im Fettgewebe und in der Leber gebildet und freigesetzt werden, sind wichtige pathogenetische Mediatoren der NA-
FLD. Das metabolische Syndrom ist die klinisch bedeutsamste Erkrankung, die durch die beschriebenen Veränderungen des Lipidstoffwechsels charakterisiert ist und mit einer NAFLD einhergeht. Auch die so genannten Lipodystrophien und primäre Hypertriglyzeridämien sind wichtige Ursachen der NAFLD.

\section{Korrespondierender Autor}

Dr. S. Bilz

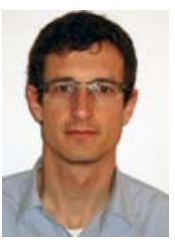

Klinik für Endokrinologie, Diabetologie und Klinische Ernährung, Universitätsspital Basel Petersgraben 4, 4031 Basel sbilz@uhbs.ch

Interessenkonflikt. Es besteht kein Interessenkonflikt. Der korrespondierende Autor versichert, dass keine Verbindungen mit einer Firma, deren Produkt in dem Artikel genannt ist, oder einer Firma, die ein Konkurrenzprodukt vertreibt, bestehen. Die Präsentation des Themas ist unabhängig und die Darstellung der Inhalte produktneutral.

\section{Literatur}

1. Angulo P (2002) Nonalcoholic fatty liver disease. $N$ Engl J Med 346: 1221-1231

2. Bilz S, Samuel V, Morino K et al. (2006) Activation of the farnesoid $X$ receptor improves lipid metabolism in combined hyperlipidemic hamsters. AJP Endocrinol Metab 290: E716-E722

3. Bugianesi E, Pagotto U, Manini R et al. (2005) Plasma adiponectin in nonalcoholic fatty liver is related to hepatic insulin resistance and hepatic fat content, not to liver disease severity. J Clin Endocrinol Metab 90: 3498-3504

4. Cha JY, Repa JJ (2007) The liver X receptor (LXR) and hepatic lipogenesis: the carbohydrate-response element-binding protein is a target gene of LXR. J Biol Chem 282: 743-751

5. Charlton M, Sreekumar R, Rasmussen D et al. (2002) Apolipoprotein synthesis in nonalcoholic steatohepatitis. Hepatology 35: 898-904

6. Chen G, Liang G, Ou J et al. (2004) Central role for liver $X$ receptor in insulin-mediated activation of Srebp-1c transcription and stimulation of fatty acid synthesis in liver. Proc Natl Acad Sci USA 101: 11.245-11.250

7. Cuchel M, Bloedon LT, Szapary PO et al. (2007) Inhibition of microsomal triglyceride transfer protein in familial hypercholesterolemia. N Engl J Med 356: 148-156

8. Dentin R, Benhamed F, Hainault I et al. (2006) Liver-specific inhibition of ChREBP improves hepatic steatosis and insulin resistance in ob/ob mice. Diabetes 55: 2159-2170

9. Diraison F, Moulin P, Beylot M (2003) Contribution of hepatic de novo lipogenesis and reesterification of plasma non esterified fatty acids to plasma triglyceride synthesis during non-alcoholic fatty liver disease. Diabetes Metab 29: 478-485 
10. Donnelly KL, Smith Cl, Schwarzenberg SJ et al. (2005) Sources of fatty acids stored in liver and secreted via lipoproteins in patients with nonalcoholic fatty liver disease. J Clin Invest 115: 13431351

11. Duane WC (1995) Abnormal bile acid absorption in familial hypertriglyceridemia. J Lipid Res 36: 96 107

12. Garg A (2004) Acquired and inherited lipodystrophies. N Engl J Med 350: 1220-1234

13. Ginsberg HN (2000) Insulin resistance and cardiovascular disease. J Clin Invest 106: 453-458

14. Holt HB, Wild SH, Wood PJ et al. (2006) Non-esterified fatty acid concentrations are independently associated with hepatic steatosis in obese subjects. Diabetologia 49: 141-148

15. Lin J, Yang R, Tarr PT et al. (2005) Hyperlipidemic effects of dietary saturated fats mediated through PGC-1 beta coactivation of SREBP. Cell 120: 261273

16. Mari M, Caballero F, Colell A et al. (2006) Mitochondrial free cholesterol loading sensitizes to TNF- and Fas-mediated steatohepatitis. Cell Metab 4: $185-198$

17. Matsumoto M, Ogawa W, Akimoto K et al. (2003) PKC lambda in liver mediates insulin-induced SREBP-1c expression and determines both hepatic lipid content and overall insulin sensitivity. J Clin Invest 112: 935-944

18. Mitro N, Mak PA, Vargas L et al. (2007) The nuclear receptor LXR is a glucose sensor. Nature 445: 219223

19. Nakamuta M, Kohjima M, Morizono S et al. (2005) Evaluation of fatty acid metabolism-related gene expression in nonalcoholic fatty liver disease. Int J Mol Med 16: 631-635

20. Partin JS, Partin JC, Schubert WK et al. (1974) Liver ultrastructure in abetalipoproteinemia: evolution of micronodular cirrhosis. Gastroenterology 67: 107-118

21. Samuel VT, Liu ZX, Qu X et al. (2004) Mechanism of hepatic insulin resistance in non-alcoholic fatty liver disease. J Biol Chem 279: 32.345-32.353

22. Schonfeld G, Patterson BW, Yablonskiy DA et al. (2003) Fatty liver in familial hypobetalipoproteinemia: triglyceride assembly into VLDL particles is af fected by the extent of hepatic steatosis. J Lipid Res 44: 470-478

23. Shimomura I, Matsuda M, Hammer RE et al. (2000) Decreased IRS-2 and increased SREBP-1c lead to mixed insulin resistance and sensitivity in livers of lipodystrophic and ob/ob mice. Mol Cell 6: 77-86

24. Ueki K, Kondo T, Tseng YH et al. (2004) Central role of suppressors of cytokine signaling proteins in hepatic steatosis, insulin resistance, and the metabolic syndrome in the mouse. Proc Natl Acad Sci USA 101: 10.422-10.427

25. Utzschneider KM, Kahn SE (2006) The role of insulin resistance in nonalcoholic fatty liver disease. J Clin Endocrinol Metab 91: 4753-4761

26. Watanabe M, Houten SM, Wang L et al. (2004) Bile acids lower triglyceride levels via a pathway involving FXR, SHP, and SREBP-1c. J Clin Invest 113: 1408-1418

27. Wolfrum C, Asilmaz E, Luca E et al. (2004) Foxa2 regulates lipid metabolism and ketogenesis in the liver during fasting and in diabetes. Nature 432: 1027-1032

28. Yamauchi T, Kamon J, Waki H et al. (2001) The fatderived hormone adiponectin reverses insulin resistance associated with both lipoatrophy and obesity. Nat Med 7: 941-946
29. Yamauchi T, Kamon J, Minokoshi Y et al. (2002) Adiponectin stimulates glucose utilization and fatty-acid oxidation by activating AMP-activated protein kinase. Nat Med 8: 1288-1295

30. Yudkin JS (2003) Adipose tissue, insulin action and vascular disease: inflammatory signals. Int J Obes Relat Metab Disord [Suppl 3] 27: 25-28

\section{September 2007}

Ludwigshafen 05.09.2007

Wiss. Symposium Diabetes mellitus

Auskunft: Frau Gaby Kneissler, Klinikum

Ludwigshafen gGmbH, Bremserstr. 79, 67063

Ludwigshafen,

Fon: 0621 - 5034111

Fax: 0621 - 5034112

kongressmc@t-online.de

Wien 13.-15.09.2007

45. Jahrestagung der Österreichischen Gesellschaft für Kinder- und

Jugendheilkunde

Themen: Endokrinologie und Diabetes

Neuropädiatrie, Schlaf, Kardiologie, Allergologie

Auskunft: Ärztezentrale Med.Info,

Helferstorferstrasse 4, 1014 Wien, Österreich

Fon: $(+43 / 1) 53116-33$

Fax: (+43/1) $53116-61$

azmedinfo@media.co.at

Berlin 26.-29.09.2007

33rd Annual Meeting of the International

Society for Pediatric and Adolescent

Diabetes

Diabetes in Motion in the Year of the Child

Auskunft: K.I.T. GmbH, Kurfürstendamm 71

10709 Berlin

Fon: 030/246 030

Fax: 030/246 03200

ispad2007@kit-group.org,

www.kit-group.org

Innsbruck 27.-29.09.2007

38.Jahrestagung der Österr. Ges. für Innere

Medizin

Themen: Update Diabetestherapie, Nosokomiale Infektionen, Venöse Thromboembolie,

Multimodale Therapie in der Onkologie

Auskunft: Ärztezentrale Med.Info,

Helferstorferstrasse 4, 1014 Wien, Österreich,

Fon: $(+43 / 1) 53116-32$

Fax: (+43/1) $53116-61$

azmedinfo@media.co.at,

www.oegim.at
Weitere Termine finden Sie im Internet unter www.DerDiabetologe.springer.de 